Article

\title{
Synthesis and Anti-Tumor Activities of 4-Anilinoquinoline Derivatives
}

\author{
Dan Liu *, Tian Luan, Jian Kong, Ying Zhang and Hai-Feng Wang \\ Received: 2 November 2015 ; Accepted: 2 December 2015 ; Published: 23 December 2015 \\ Academic Editor: D. Hadjipavlou-Litina \\ Department of Pharmaceutical Engineering, College of Parmaceutical and Biological Engineering, \\ Shenyang University of Chemical Technology, Shenyang 110142, China; sapphire0614@163.com (T.L.); \\ kongjianwm2006@163.com (J.K.); zhingky621@126.com (Y.Z.); 38whf@163.com (H.-F.W.) \\ * Correspondence: liudan20040318@163.com; Tel./Fax: +86-24-8938-3906
}

\begin{abstract}
Twenty-two 7-fluoro (or 8-methoxy)-4-anilinoquinolines compounds were designed and synthesized as potentially potent and selective antitumor inhibitors. All the prepared compounds were evaluated for their in vitro antiproliferative activities against the HeLa and BGC823 cell lines. Ten compounds (1a-g; $\mathbf{2} \mathbf{c} ; \mathbf{2} \mathbf{e}$ and $\mathbf{2} \mathbf{i})$ exhibited excellent antitumor activity superior to that of gefitinib. Among the ten compounds; seven (1a-c; $\mathbf{1 e - 1} \mathbf{g}$ and $\mathbf{2 i})$ displayed excellent selectivity for BGC 823 cells. In particular; $1 \mathbf{f}$ and $2 \mathbf{i}$ exhibited potent cytotoxic activities against HeLa cells and BGC823 cells with better $\mathrm{IC}_{50}$ values than gefitinib.
\end{abstract}

Keywords: 4-anilinoquinolines; EGFR; antitumor; inhibitor

\section{Introduction}

The epidermal growth factor receptor (EGFR) and its closely related family member HER2 play a critical role in mediating growth factor signaling, which make them interesting drug targets for oncology. EGFR and HER2 have been extensively investigated, and various classes of small molecule kinase inhibitors have emerged as promising strategies to inhibited EGFR and HER2 kinase activity.

A considerable number of 4-anilinoquinazoline-based kinase inhibitors are known, including gefitinib (1), erlotinib (2) [1,2], lapatinib (3) [3,4], and afatinib (4) [5,6] (Figure 1). Previous SAR studies suggest that the quinazoline core was the best scaffold for the development of EGFR inhibitors and the quinazoline N3 interacts with the kinase domain via a water-mediated hydrogen bond to the side chain of the gatekeeper Thr790 of EGFR [7,8]. The quinazoline-based kinase inhibitors are widely used in medicinal chemistry and chemical biology research. For instance, Some C2 position modification of quinazoline-based compounds resulted in potent cytotoxic activities $[9,10]$. On the basis of the SAR studies of quinazolines, a series of compounds were developed where the N3 of the quinazoline was replaced by a C-CN group, such as neratinib (5, Figure 1) [11,12]. However, Rauh et al. have found that there was no evidence with the existence of a water molecule mediating the binding of N3 of the quinazoline core to the side chain of Thr790 by calculating the corresponding electron density maps. Quinolines 6 and 7 (Figure 1) were found to be highly active kinase inhibitors in biochemical assays and were further investigated for their biological effect on EGFR-dependent $\mathrm{Ba} / \mathrm{F} 3$ cells and non-small cell lung cancer (NSCLC) cell lines [13]. Furthermore, studies have shown that the 4-anilino group can interact with the hydrophobic pocket of EGFR, the introduction of electron-donating groups in benzene ring of 4-anilinoquinazoline can increase the density of electron cloud on the quinazoline N1, and follow by increase the interaction with EGFR [14].

The aforementioned findings stimulated our interest in designing and synthesizing a series of 7-fluoro or 8-methoxy 4-anilinoquinolines which were acticipated to be as potent as their quinazoline 
counterparts. The activity of the target compounds were evaluated by human cervical cancer cell line (HeLa) and human gastric carcinoma cell line (BGC-823), and both of the cell lines had been proved to be highly expressed cell line of EGFR $[15,16]$.<smiles>COc1cc2ncnc(Nc3ccc(F)c(Cl)c3)c2cc1OCCCN1CCOCC1</smiles>

Gefitinib 1<smiles>C#Cc1cccc(Nc2ncnc3cc(OCCOC)c(OCCOC)cc23)c1</smiles>

Erlotinib 2<smiles>CS(=O)(=O)CNCc1ccc(-c2ccc3ncnc(Nc4ccc(OCc5ccc(F)cc5)c(Cl)c4)c3c2)o1</smiles>

Lapatinib 3<smiles>CN(C)C/C=C/C(=O)Nc1cc2c(Nc3ccc(F)c(Cl)c3)ncnc2cc1OC1CCOC1</smiles>

Afatinib 4<smiles>C=CC(=O)Nc1ccc2nccc(Nc3cccc(Br)c3)c2c1</smiles>

6<smiles>CCOc1cc2ncc(N)c(Nc3ccc(OCc4ccccn4)c(Cl)c3)c2cc1NC(=O)/C=C/CN(C)C</smiles>

Neratinib 5<smiles>CN(C)CCCC(=O)Nc1ccc2nccc(Nc3cccc(Br)c3)c2c1</smiles>

Figure 1. Several inhibitors of EGFR tyrosine kinases.

\section{Results and Discussion}

\subsection{Chemistry}

The target compounds $\mathbf{1 a}-\mathbf{h}$ and $\mathbf{2} \mathbf{a}-\mathbf{n}$ were synthesized via a convenient six-step reaction depicted in Scheme 1 and the respective experimental details are given in Section 3.1. Twenty-two compounds were obtained and their MS, ${ }^{1} \mathrm{H}-\mathrm{NMR}$ spectroscopy data are provided in Section 3.1.<smiles>[Y]c1cccc(N)c1[Y]</smiles>

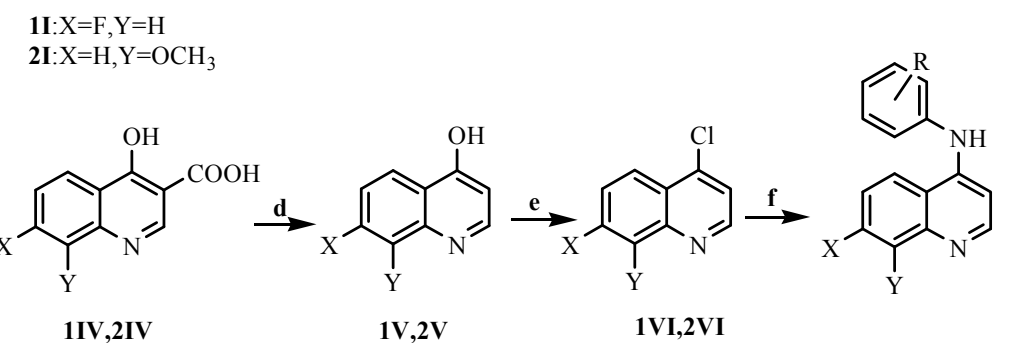

Scheme 1. General procedure for the synthesis of target compounds $\mathbf{1 a}-\mathbf{h}$ and $\mathbf{2 a}-\mathbf{n}$. Reagents and conditions: (a) EtOH, reflux; (b) Dowtherm A, 250-260 ${ }^{\circ} \mathrm{C}$; (c) $10 \% \mathrm{NaOH}, \mathrm{EtOH}$, reflux; (d) Dowtherm A, $240{ }^{\circ} \mathrm{C}$; (e) $\mathrm{POCl}_{3}$; (f) $i$-PrOH, pyridine- $\mathrm{HCl}$, substituted aniline, reflux. 


\subsection{Biological Evaluation}

The biological activities of all the target compounds $\mathbf{1} \mathbf{a}-\mathbf{h}$ and $\mathbf{2} \mathbf{a}-\mathbf{n}$ were evaluated in vitro by MTT assay against HeLa and BGC-823 cell lines with gefitinib (1) as the positive control. Their inhibition rate and $\mathrm{IC}_{50}$ values are listed in Table 1.

Table 1. Antiproliferactive activity of the target compounds on HeLa and BGC-823 cell lines.

\begin{tabular}{|c|c|c|c|c|c|}
\hline \multirow{2}{*}{ Compound } & \multirow{2}{*}{ Substituents } & \multicolumn{2}{|c|}{ Inhibition Rate $\%^{a}$} & \multicolumn{2}{|c|}{$\mathrm{IC}_{50}(\mu \mathrm{M})^{\mathrm{b}}$} \\
\hline & & HeLa & BGC-823 & HeLa & BGC-823 \\
\hline $1 \mathbf{a}$ & $\mathrm{X}=\mathrm{F}, \mathrm{Y}=\mathrm{H}, \mathrm{R}=3^{\prime}-\mathrm{Cl}$ & 46.9 & 77.5 & 17.29 & 3.63 \\
\hline $1 \mathrm{~b}$ & $\mathrm{X}=\mathrm{F}, \mathrm{Y}=\mathrm{H}, \mathrm{R}=4^{\prime}-\mathrm{Cl}$ & 40.3 & 63.1 & 20.39 & 7.83 \\
\hline 1c & $\mathrm{X}=\mathrm{F}, \mathrm{Y}=\mathrm{H}, \mathrm{R}=3^{\prime}-\mathrm{F}$ & 37.0 & 65.3 & 19.82 & 9.10 \\
\hline $1 d$ & $\mathrm{X}=\mathrm{F}, \mathrm{Y}=\mathrm{H}, \mathrm{R}=4^{\prime}-\mathrm{F}$ & 20.4 & 49.5 & 43.25 & 11.10 \\
\hline 1e & $\mathrm{X}=\mathrm{F}, \mathrm{Y}=\mathrm{H}, \mathrm{R}=3^{\prime}-\mathrm{Cl}, 4^{\prime}-\mathrm{Cl}$ & 40.8 & 56.9 & 36.69 & 8.29 \\
\hline 1f & $\mathrm{X}=\mathrm{F}, \mathrm{Y}=\mathrm{H}, \mathrm{R}=3^{\prime}-\mathrm{Cl}, 4^{\prime}-\mathrm{F}$ & 46.8 & 67.5 & 10.18 & 8.32 \\
\hline $1 \mathrm{~g}$ & $\mathrm{X}=\mathrm{F}, \mathrm{Y}=\mathrm{H}, \mathrm{R}=4^{\prime}-\mathrm{CH}_{3}$ & 41.7 & 59.4 & 18.69 & 7.08 \\
\hline $1 \mathrm{~h}$ & $\mathrm{X}=\mathrm{F}, \mathrm{Y}=\mathrm{H}, \mathrm{R}=4^{\prime}-\mathrm{OCH}_{3}$ & 36.1 & 35.1 & 55.76 & 21.61 \\
\hline $2 a$ & $\mathrm{X}=\mathrm{H}, \mathrm{Y}=\mathrm{OCH}_{3}, \mathrm{R}=3^{\prime}-\mathrm{Cl}$ & 35.3 & 19.5 & 66.69 & $>10$ \\
\hline $2 b$ & $\mathrm{X}=\mathrm{H}, \mathrm{Y}=\mathrm{OCH}_{3}, \mathrm{R}=4^{\prime}-\mathrm{Cl}$ & 31.5 & 13.1 & $>10$ & $>10$ \\
\hline $2 c$ & $\mathrm{X}=\mathrm{H}, \mathrm{Y}=\mathrm{OCH}_{3}, \mathrm{R}=3^{\prime}-\mathrm{F}$ & 31.9 & 48.1 & 75.87 & 11.67 \\
\hline $2 d$ & $\mathrm{X}=\mathrm{H}, \mathrm{Y}=\mathrm{OCH}_{3}, \mathrm{R}=4^{\prime}-\mathrm{F}$ & 18.9 & 37.5 & $>10$ & 46.41 \\
\hline $2 \mathbf{e}$ & $\mathrm{X}=\mathrm{H}, \mathrm{Y}=\mathrm{OCH}_{3}, \mathrm{R}=3^{\prime}-\mathrm{Cl}, 4^{\prime}-\mathrm{Cl}$ & 30.8 & 48.7 & 45.87 & 11.45 \\
\hline $2 \mathrm{f}$ & $\mathrm{X}=\mathrm{H}, \mathrm{Y}=\mathrm{OCH}_{3}, \mathrm{R}=3^{\prime}-\mathrm{Cl}, 4^{\prime}-\mathrm{F}$ & 37.2 & 20.2 & 45.84 & $>10$ \\
\hline $2 \mathrm{~g}$ & $\mathrm{X}=\mathrm{H}, \mathrm{Y}=\mathrm{OCH}_{3}, \mathrm{R}=4^{\prime}-\mathrm{CH}_{3}$ & 30.5 & 31.1 & 99.54 & 61.52 \\
\hline $2 \mathrm{~h}$ & $\mathrm{X}=\mathrm{H}, \mathrm{Y}=\mathrm{OCH}_{3}, \mathrm{R}=4^{\prime}-\mathrm{OCH}_{3}$ & 21.9 & 32.0 & $>10$ & 74.60 \\
\hline $2 \mathbf{i}$ & $\mathrm{X}=\mathrm{H}, \mathrm{Y}=\mathrm{OCH}_{3}, \mathrm{R}=4^{\prime}-\mathrm{CH}\left(\mathrm{CH}_{3}\right)_{2}$ & 59.1 & 78.4 & 7.15 & 4.65 \\
\hline $2 j$ & $\mathrm{X}=\mathrm{H}, \mathrm{Y}=\mathrm{OCH}_{3}, \mathrm{R}=3^{\prime}-\mathrm{Cl}, 4^{\prime}-\mathrm{CH}_{3}$ & 32.7 & 24.5 & 71.26 & $>10$ \\
\hline $2 \mathbf{k}$ & $\mathrm{X}=\mathrm{H}, \mathrm{Y}=\mathrm{OCH}_{3}, \mathrm{R}=2^{\prime} \mathrm{F}, 3^{\prime}-\mathrm{F}, 4^{\prime}-\mathrm{F}$ & 32.5 & 27.6 & 57.88 & $>10$ \\
\hline 21 & $\mathrm{X}=\mathrm{H}, \mathrm{Y}=\mathrm{OCH}_{3}, \mathrm{R}=4^{\prime}-\mathrm{NO}_{2}$ & 35.8 & 36.8 & 13.48 & $>10$ \\
\hline $2 m$ & $\mathrm{X}=\mathrm{H}, \mathrm{Y}=\mathrm{OCH}_{3}, \mathrm{R}=4^{\prime}-\mathrm{OH}$ & 32.7 & 25.6 & 39.58 & $>10$ \\
\hline $2 n$ & $\mathrm{X}=\mathrm{H}, \mathrm{Y}=\mathrm{OCH}_{3}, \mathrm{R}=3^{\prime}-\mathrm{CN}$ & 28.6 & 16.0 & 30.61 & $>10$ \\
\hline Gefitinib & & 42.6 & 46.0 & 17.12 & 19.27 \\
\hline
\end{tabular}

${ }^{\mathrm{a}}$ Inhibitory percentage of cells treated with each compound at a concentration of $10 \mu \mathrm{M}$ for $96 \mathrm{~h}^{\mathrm{b}}{ }^{\mathrm{b}}$ The agent concentration that inhibited HeLa and BGC-823 cells growth by $50 \%$.

As shown in Table 1, 7-fluoro-4-anilinoquinolines $\mathbf{1 a - g}$ displayed better cytotoxic activities against BGC-823 cells than HeLa cells and exhibited $\mathrm{IC}_{50}$ values within the $3.63-11.10 \mu \mathrm{mol} / \mathrm{L}$ range (on $\mathrm{BGC}-823$ cells). It seems that substituent changes on the quinoline ring at the $\mathrm{C} 7$ or C8 carbons have a great influence on the antiproliferactive activity. In general, 7-fluoro-4-anilinoquinolines of these twenty-two compounds, were more active than those of the corresponding 8-methoxy-4-anilinoquinolines, as demonstrated by comparison of $\mathbf{1} \mathbf{a}-\mathbf{h}$ and $\mathbf{2} \mathbf{a}-\mathbf{h}$. Thirdly, it was found that substituent changes on the benzene ring had little influence on antiprolifeactive activity, whether it was electron-donating or electron-withdrawing substituent at any position (1a vs. 1b and 1d; $\mathbf{2 g}$ vs. $\mathbf{2 b}$ and $\mathbf{2 d} ; \mathbf{2 g}$ and $\mathbf{2 m}$ vs. $\mathbf{2 l}$ and $\mathbf{2 n}$ ). Lastly, most of the 8-methoxy-4-anilinoquinolines exhibited moderate antiproliferactive activity against the HeLa and BGC-823 cell lines, except for compound $\mathbf{2} \mathbf{i}$. It was noteworthy that compound $\mathbf{2} \mathbf{i}$ bearing a methoxy group on the quinoline ring and an isopropyl group on the benzene ring showed remarkable inhibitory effects on HeLa cells and BGC-823 cells $\left(\mathrm{IC}_{50}=7.15 \mu \mathrm{M}, \mathrm{IC}_{50}=4.65 \mu \mathrm{M}\right)$, which represented a 2.4 and 4.1 -fold increase in antitumor activity compared to gefitinib $\left(\mathrm{IC}_{50}=17.12 \mu \mathrm{M}\right.$ against HeLa cells, $\mathrm{IC}_{50}=19.27 \mu \mathrm{M}$ against BGC-823 cells), respectively. 


\section{Experimental Section}

\subsection{Chemistry}

All reagents and solvents were commercially available and were used without further purification. The melting points were determined on an electrically heated X-4 digital visual melting point apparatus (Tech, Beijing, China) and were uncorrected. ${ }^{1} \mathrm{H}-\mathrm{NMR}$ spectra were recorded on an ARX-300 or AV-600 spectrometer (Bruker, Fällanden, Switzerland) at room temperature, and chemical shifts were measured in ppm downfield from TMS as internal standard. Mass spectra were recorded on Thermo-Finnigan LCQ equipment (ThermoFinnigan, San Francisco, CA, USA) with the positive Electron Spray Ionization (ESI) mode and are reported as $m / z$. Analytical thin layer chromatography (TLC) on silica gel plates containing UV indicator (YuHua, Gongyi, China) was routinely employed to follow the course of reactions and to check the purity of products.

\subsubsection{Preparation of the Intermediates 1II-1VI}

Diethyl (ethoxymethylene) malonate $(6.48 \mathrm{~g}, 30.00 \mathrm{mmol})$ and $m$-fluroaniline $(2.78 \mathrm{~g}, 25.00 \mathrm{mmol})$ was stirred in refluxing ethanol $(10 \mathrm{~mL})$ for $1.5 \mathrm{~h}$. The mixture was concentrated under reduced pressure. The residue was crystallized in petroleum ether, filtered off, and air-dried to get 1II. Compound 1II (2.80 g, $9.96 \mathrm{mmol})$ was stirred in Dowtherm-A (8 mL) for $0.5 \mathrm{~h}$ at $260{ }^{\circ} \mathrm{C}$. After the reaction was over by TLC, the mixture was cooled to room temperature and petroleum ether was added to get the crude ester, which was further washed with petroleum ether to afford 1III, as a white solid. 1III was hydrolyzed in refluxing $\mathrm{NaOH}$ solution $(10 \%, 20 \mathrm{~mL})$ for $1.5 \mathrm{~h}$ to give 1IV. Compound 1IV $(1.24 \mathrm{~g}, 6 \mathrm{mmol})$ was stirred in Dowtherm-A $(10 \mathrm{~mL})$ for $1 \mathrm{~h}$ at $240{ }^{\circ} \mathrm{C}$. After the reaction was over as monitored by TLC, the reaction mixture was cooled to room temperature and the crude was washed with petroleum ether to afford $\mathbf{1 V}$ as a white solid. To a solution of $\mathbf{1 V}$ $(1.20 \mathrm{~g}, 7.36 \mathrm{mmol})$ in 1,2-dichloroethane $(30 \mathrm{~mL}), \mathrm{POCl}_{3}(1.35 \mathrm{~g}, 8.83 \mathrm{mmol})$ was added dropwise. The mixture was refluxed for $1 \mathrm{~h}$. Saturated $\mathrm{NaHCO}_{3}$ solution was added to neutralize the reaction mixture, which was worked up with 1,2-dichloroethane. The organic layer was dried over anhydrous $\mathrm{MgSO}_{4}$, filtered and concentrated in vacuo. The residue was purified by column chromatography (silica gel) using petroleum ether/ethyl acetate as an eluent (5:1) to produce 1VI as a white solid [17]. 1VI: ${ }^{1} \mathrm{H}-\mathrm{NMR}\left(300 \mathrm{MHz}, \mathrm{CDCl}_{3}\right.$ ): 8.79-8.78 (d, 2-H, 1H), 8.27-8.24 (q, 5-H, 1H), 7.77-7.75 (q, 8-H, 1H), 7.47-7.46 (d, 3-H, 1H), 7.44-7.42 (q, 6-H, 1H), ESI-MS $(m / z): 182.4[\mathrm{M}+\mathrm{H}]^{+}$.

\subsubsection{Preparation of the Intermediates 2II-2VI}

Compounds 2II-2VI were prepared in the same manner as 2II-2VI. 2VI: ${ }^{1} \mathrm{H}-\mathrm{NMR}(300 \mathrm{MHz}$, $\left.\mathrm{CDCl}_{3}\right): 4.11\left(3 \mathrm{H}, \mathrm{s}, \mathrm{OCH}_{3}\right), 7.11(1 \mathrm{H}, \mathrm{d}, J=7.2 \mathrm{~Hz}, \mathrm{Ar}-\mathrm{H}-7), 7.53-7.60(2 \mathrm{H}, \mathrm{m}, \mathrm{Ar}-\mathrm{H}-3,6)$, $7.81(1 \mathrm{H}, \mathrm{dd}, J=8.7 \mathrm{~Hz}, J=1.2 \mathrm{~Hz}, \mathrm{Ar}-\mathrm{H}-5), 8.80(1 \mathrm{H}, \mathrm{d}, J=4.8 \mathrm{~Hz}, \mathrm{Ar}-\mathrm{H}-2)$; ESI-MS $(m / z)$ : $194.4[\mathrm{M}+\mathrm{H}]^{+}$.

\subsubsection{Preparation of the Title Compounds $\mathbf{1}$ and $\mathbf{2}$}

A mixture of compound 1VI $(0.040 \mathrm{~g}, 0.22 \mathrm{mmol}), m$-chloroaniline $(0.036 \mathrm{~g}, 0.31 \mathrm{mmol})$ and pyridine hydrochloride was heated at reflux for $45 \mathrm{~min}$ in isopropanol $(6 \mathrm{~mL})$, after the reaction is over by TLC, it was cooled to room temperature and the petroleum ether $(4 \mathrm{~mL})$ and $\mathrm{NaHCO}_{3}(10 \mathrm{~mL})$ were added into the reaction mixture. The product was filtered and recrystallised from ethanol to give the title compound $\mathbf{1}$. Compound $\mathbf{2}$ was prepared in the same manner as $\mathbf{1}$.

4-(3'-Chlorophenylamino)-7-fluoroquinoline (1a). Yield: 96.25\%; white solid. m.p. 196-197 ${ }^{\circ} \mathrm{C} ;{ }^{1} \mathrm{H}-\mathrm{NMR}$ $\left(600 \mathrm{MHz} \mathrm{CDCl}_{3}\right) \delta: 8.62(\mathrm{~d}, J=4.8 \mathrm{~Hz}, 1 \mathrm{H}, \mathrm{ArH}), 7.94(\mathrm{dd}, J=9.0,6.0 \mathrm{~Hz}, 1 \mathrm{H}, \mathrm{ArH}), 7.71(\mathrm{dd}, J=4.2$, $2.4 \mathrm{~Hz}, 1 \mathrm{H}, \mathrm{ArH}), 7.37-7.27$ (m, 3H, ArH), 7.19-7.17 (m, 2H, ArH), 7.01 (d, 1H, J = 5.4 Hz, ArH). $\operatorname{ESI-MS}(m / z): 273.4[\mathrm{M}+\mathrm{H}]^{+}$. 
4-(4'-Chlorophenylamino)-7-fluoroquinoline (1b). Yield: 92.93\%. m.p. $194-195{ }^{\circ} \mathrm{C},{ }^{1} \mathrm{H}-\mathrm{NMR}(600 \mathrm{MHz}$, $\left.\mathrm{CDCl}_{3}\right) \delta: 8.57(\mathrm{~s}, 1 \mathrm{H}, \mathrm{ArH}), 7.96(\mathrm{dd}, J=8.4,6.0 \mathrm{~Hz}, 1 \mathrm{H}, \mathrm{ArH}), 7.70(\mathrm{~d}, 1 \mathrm{H}, J=10.2 \mathrm{~Hz}, \mathrm{ArH})$, 7.41-7.40 (m, 2H, ArH), 7.31 (t, J = 8.4 Hz, 1H, ArH), 7.27-7.25 (m, 2H, ArH), $6.90(\mathrm{~d}, J=4.8 \mathrm{~Hz}, 1 \mathrm{H}$, $\operatorname{ArH})$. ESI-MS $(m / z): 273.4[\mathrm{M}+\mathrm{H}]^{+}$.

4-(3'-Fluorophenylamino)-7-fluoroquinoline (1c). Yield: 95.37\%; m.p. 173-174 ${ }^{\circ} \mathrm{C} ;{ }^{1} \mathrm{H}-\mathrm{NMR}(600 \mathrm{MHz}$, $\left.\mathrm{CDCl}_{3}\right) \delta: 8.62(\mathrm{~s}, 1 \mathrm{H}, \mathrm{ArH}), 7.94(\mathrm{dd}, J=9.0,6.0 \mathrm{~Hz}, 1 \mathrm{H}, \mathrm{ArH}), 7.70(\mathrm{dd}, J=6.6,2.1 \mathrm{~Hz}, 1 \mathrm{H}, \mathrm{ArH}), 7.38$ $(\mathrm{dd}, J=14.7,8.1 \mathrm{~Hz}, 1 \mathrm{H}, \mathrm{ArH}), 7.34-7.31(\mathrm{~m}, 1 \mathrm{H}, 1 \mathrm{H}, \mathrm{ArH}), 7.06(\mathrm{t}, J=4.5 \mathrm{~Hz}, 1 \mathrm{H}, \mathrm{ArH}), 7.04-7.02$ (m, 2H, ArH), 6.91-6.88 (m, 1H, ArH). ESI-MS $(m / z): 257.4[\mathrm{M}+\mathrm{H}]^{+}$.

4-(4'-Fluorophenylamino)-7-fluoroquinoline (1d). Yield: 93.66\%; m.p. 178-179 ${ }^{\circ} \mathrm{C} ;{ }^{1} \mathrm{H}-\mathrm{NMR}(600 \mathrm{MHz}$, $\left.\mathrm{CDCl}_{3}\right) \delta: 8.54-8.53(\mathrm{~s}, 1 \mathrm{H}, \mathrm{ArH}), 7.94(\mathrm{~d}, J=6.0 \mathrm{~Hz}, 1 \mathrm{H}, \mathrm{ArH}), 7.68(\mathrm{dd}, J=10.2,3.0 \mathrm{~Hz}, 1 \mathrm{H}$, ArH), 7.32-7.28 (m, 3H, ArH), 7.17-7.13 (m, 2H, ArH), $6.74(\mathrm{~d}, J=5.4 \mathrm{~Hz} 1 \mathrm{H}, \mathrm{ArH})$. ESI-MS $(m / z)$ : $257.4[\mathrm{M}+\mathrm{H}]^{+}$.

4-(3'-Chloro-4'-chlorophenylamino)-7-fluoroquinoline (1e). Yield: 91.34\%; m.p. 191-192 ${ }^{\circ} \mathrm{C} ;{ }^{1} \mathrm{H}-\mathrm{NMR}$ $\left(600 \mathrm{MHz}, \mathrm{CDCl}_{3}\right) \delta: 8.61(\mathrm{~d}, J=4.8 \mathrm{~Hz}, 1 \mathrm{H}, \mathrm{ArH}), 7.97(\mathrm{dd}, J=9.0,6.0 \mathrm{~Hz}, 1 \mathrm{H}, \mathrm{ArH}), 7.71(\mathrm{dd}, J=9.6$, $2.4 \mathrm{~Hz}, 1 \mathrm{H}, \mathrm{ArH}), 7.48(\mathrm{~d}, J=9.0 \mathrm{~Hz}, 1 \mathrm{H}, \mathrm{ArH}), 7.41(\mathrm{~d}, J=2.4 \mathrm{~Hz}, 1 \mathrm{H}, \mathrm{ArH}), 7.34-7.27(\mathrm{~m}, 1 \mathrm{H}, \mathrm{ArH})$, $7.16(\mathrm{dd}, J=8.7,2.7 \mathrm{~Hz}, 1 \mathrm{H}, \mathrm{ArH}), 6.97(\mathrm{dd}, J=5.4 \mathrm{~Hz}, 1 \mathrm{H}, \operatorname{ArH})$. ESI-MS $(m / z): 307.3[\mathrm{M}+\mathrm{H}]^{+}$.

4-( $3^{\prime}$-Chloro-4'-fluoroamino)-7-fluoroquinoline (1f). Yield: 74.72\%; m.p. 193-194 ${ }^{\circ} \mathrm{C} ;{ }^{1} \mathrm{H}-\mathrm{NMR}(600 \mathrm{MHz}$, $\left.\mathrm{CDCl}_{3}\right) \delta: 8.58(\mathrm{~s}, 1 \mathrm{H}, \mathrm{ArH}), 7.93(\mathrm{dd}, J=9.0,5.4 \mathrm{~Hz}, 1 \mathrm{H}, \mathrm{ArH}), 7.70(\mathrm{dd}, J=10.2,2.4 \mathrm{~Hz}, 1 \mathrm{H}, \mathrm{ArH})$, $7.38(\mathrm{dd}, J=10.0,2.4 \mathrm{~Hz}, 1 \mathrm{H}, \mathrm{ArH}), 7.34-7.31(\mathrm{~m}, 1 \mathrm{H}, \mathrm{ArH}), 7.27-7.19(\mathrm{~m}, 2 \mathrm{H}, \mathrm{ArH}), 6.82(\mathrm{~d}, J=5.4 \mathrm{~Hz}$, 1H, ArH). ESI-MS $(m / z): 291.4[\mathrm{M}+\mathrm{H}]^{+}$.

4-(4'-Methylphenylamino)-7-fluoroquinoline (1g). Yield: 96.56\%, m.p. 171-172 ${ }^{\circ} \mathrm{C} ;{ }^{1} \mathrm{H}-\mathrm{NMR}(600 \mathrm{MHz}$, $\left.\mathrm{CDCl}_{3}\right) \delta: 8.50(\mathrm{~d}, J=5.4 \mathrm{~Hz}, 1 \mathrm{H}, \mathrm{ArH}), 7.96(\mathrm{dd}, J=9.3,5.7 \mathrm{~Hz}, 1 \mathrm{H}, \mathrm{ArH}), 7.68(\mathrm{dd}, J=10.2,2.4 \mathrm{~Hz}$, $1 \mathrm{H}, \mathrm{ArH}), 7.31-7.27(\mathrm{~m}, 1 \mathrm{H}, \mathrm{ArH}), 7.26-7.25(\mathrm{~m}, 2 \mathrm{H}, \mathrm{ArH}), 7.22-7.20(\mathrm{~m}, 2 \mathrm{H}, \mathrm{ArH}), 6.83(\mathrm{~d}, J=4.2 \mathrm{~Hz}$, $1 \mathrm{H}, \mathrm{ArH}), 2.40\left(\mathrm{~s}, 3 \mathrm{H}, \mathrm{CH}_{3}\right)$. ESI-MS $(m / z): 253.4[\mathrm{M}+\mathrm{H}]^{+}$.

4-( $4^{\prime}$-Methoxyphenylamino)-7-fluoroquinoline (1h). Yield: 76.15\%, m.p. $181-182{ }^{\circ} \mathrm{C} ;{ }^{1} \mathrm{H}-\mathrm{NMR}(600 \mathrm{MHz}$, $\left.\mathrm{CDCl}_{3}\right) \delta: 8.46(\mathrm{~d}, J=5.4 \mathrm{~Hz}, 1 \mathrm{H}, \mathrm{ArH}), 7.98(\mathrm{dd}, J=9.0,5.4 \mathrm{~Hz}, 1 \mathrm{H}, \mathrm{ArH}), 7.68(\mathrm{dd}, J=9.9,2.7 \mathrm{~Hz}$, 1H, ArH), 7.30-7.27 (m, 1H, ArH), 7.26-7.25 (m, 2H, ArH), 7.00-6.98 (m, 2H, ArH), $6.65(\mathrm{~d}, J=5.4 \mathrm{~Hz}$, $1 \mathrm{H}, \mathrm{ArH}) \cdot 3.88\left(\mathrm{~s}, 3 \mathrm{H}, \mathrm{OCH}_{3}\right)$; ESI-MS ( $\left.m / z\right): 269.3[\mathrm{M}+\mathrm{H}]^{+}$.

4-(3'-Chlorophenylamino)-8-methoxyquinoline (2a). Yield: 76.16\%, m.p. $250-251{ }^{\circ} \mathrm{C} ;{ }^{1} \mathrm{H}-\mathrm{NMR}(300 \mathrm{MHz}$, $\left.\mathrm{CDCl}_{3}\right) \delta: 8.58(\mathrm{~d}, J=5.4 \mathrm{~Hz}, 1 \mathrm{H}, \mathrm{ArH}), 7.45-7.50(\mathrm{~m}, 2 \mathrm{H}, \mathrm{ArH}), 7.40-7.30(\mathrm{~m}, 1 \mathrm{H}, \mathrm{ArH}), 7.14-7.10$ $(\mathrm{m}, 2 \mathrm{H}, \mathrm{ArH}), 7.06-6.98(\mathrm{~m}, 2 \mathrm{H}, \mathrm{ArH}), 6.88(\mathrm{~d}, J=5.4 \mathrm{~Hz}, 1 \mathrm{H}, \mathrm{ArH}), 4.08\left(\mathrm{~s}, 3 \mathrm{H}, \mathrm{OCH}_{3}\right) ; \mathrm{ESI}-\mathrm{MS}(\mathrm{m} / \mathrm{z})$ : $285.5[\mathrm{M}+\mathrm{H}]^{+}$.

4-(4'-Chlorophenylamino)-8-methoxyquinoline (2b). Yield: 77.52\%; m.p. $254-258{ }^{\circ} \mathrm{C} .{ }^{1} \mathrm{H}-\mathrm{NMR}(300 \mathrm{MHz}$, $\left.\mathrm{CDCl}_{3}\right)$ 8: $8.60(\mathrm{~d}, J=5.1 \mathrm{~Hz}, 1 \mathrm{H}, \mathrm{ArH}), 7.49-7.52(\mathrm{~m}, 2 \mathrm{H}, \mathrm{ArH}), 7.40-7.30(\mathrm{~m}, 2 \mathrm{H}, \mathrm{ArH}), 7.14-7.10(\mathrm{~m}$, $1 \mathrm{H}, \mathrm{ArH}), 7.06-6.98(\mathrm{~m}, 2 \mathrm{H}, \mathrm{ArH}), 6.84(\mathrm{~d}, J=5.1 \mathrm{~Hz}, 1 \mathrm{H}, \mathrm{ArH}), 4.08\left(\mathrm{~s}, 3 \mathrm{H}, \mathrm{OCH}_{3}\right)$; ESI-MS $(\mathrm{m} / \mathrm{z})$ : $285.5[\mathrm{M}+\mathrm{H}]^{+}$.

4-(3'-Fluorophenylamino)-8-methoxyquinoline (2c). Yield: 75.06\%; m.p. $267-269{ }^{\circ} \mathrm{C} .{ }^{1} \mathrm{H}-\mathrm{NMR}(300 \mathrm{MHz}$, $\left.\mathrm{CDCl}_{3}\right) \delta: 8.65(\mathrm{~s}, 1 \mathrm{H}, \mathrm{ArH}), 7.49-7.44(\mathrm{~m}, 2 \mathrm{H}, \mathrm{ArH}), 7.39-7.31(\mathrm{~m}, 1 \mathrm{H}, \mathrm{ArH}), 7.15(\mathrm{dd}, J=5.1$, $2.1 \mathrm{~Hz}, 1 \mathrm{H}, \mathrm{ArH}), 7.08-6.99(\mathrm{~m}, 3 \mathrm{H}, \mathrm{ArH}), 6.88-6.82(\mathrm{~m}, 1 \mathrm{H}, \mathrm{ArH}), 4.08\left(\mathrm{~s}, 3 \mathrm{H}, \mathrm{OCH}_{3}\right)$; ESI-MS $(\mathrm{m} / \mathrm{z})$ : $269.3[\mathrm{M}+\mathrm{H}]^{+}$.

4-(4'-Fluorophenylamino)-8-methoxyquinoline (2d). Yield: 84.59\%; m.p. $256-258^{\circ} \mathrm{C} .{ }^{1} \mathrm{H}-\mathrm{NMR}(300 \mathrm{MHz}$, $\left.\mathrm{CDCl}_{3}\right) \delta: 8.56(\mathrm{~d}, J=5.1 \mathrm{~Hz}, 1 \mathrm{H}, \mathrm{ArH}), 7.52-7.41(\mathrm{~m}, 2 \mathrm{H}, \mathrm{ArH}), 7.30-7.27(\mathrm{~m}, 2 \mathrm{H}, \mathrm{ArH}), 7.15-7.05$ $(\mathrm{m}, 3 \mathrm{H}, \mathrm{ArH}), 6.82(\mathrm{~d}, J=5.1 \mathrm{~Hz}, 1 \mathrm{H}, \mathrm{ArH}), 4.08\left(\mathrm{~s}, 3 \mathrm{H}, \mathrm{OCH}_{3}\right)$; ESI-MS $(m / z): 269.3[\mathrm{M}+\mathrm{H}]^{+}$.

4-( $3^{\prime}$-Chloro-4'-chlorophenylamino)-8-methoxyquinoline (2e). Yield: 79.18\%; m.p. $244-246{ }^{\circ} \mathrm{C} .{ }^{1} \mathrm{H}-\mathrm{NMR}$ $\left(300 \mathrm{MHz}, \mathrm{CDCl}_{3}\right) \delta: 8.63(\mathrm{~d}, J=5.1 \mathrm{~Hz}, 1 \mathrm{H}, \mathrm{ArH}), 7.44-7.49(\mathrm{~m}, 3 \mathrm{H}, \mathrm{ArH}), 7.41(\mathrm{~d}, J=2.4 \mathrm{~Hz}, 1 \mathrm{H}$, 
$\operatorname{ArH}), 7.16(\mathrm{dd}, J=8.7,2.4 \mathrm{~Hz}, 1 \mathrm{H}, \mathrm{ArH}), 7.08(\mathrm{~d}, J=8.4 \mathrm{~Hz}, 1 \mathrm{H}, \mathrm{ArH}), 7.03(\mathrm{~d}, J=5.1 \mathrm{~Hz}, 1 \mathrm{H}, \mathrm{ArH})$, $4.08\left(\mathrm{~s}, 3 \mathrm{H}, \mathrm{OCH}_{3}\right)$; ESI-MS $(m / z): 319.3[\mathrm{M}+\mathrm{H}]^{+}$.

4-( $3^{\prime}$-Chloro-4'-fluorophenylamino)-8-methoxyquinoline (2f). Yield: 80.14\%; m.p. $247-249{ }^{\circ} \mathrm{C} .{ }^{1} \mathrm{H}-\mathrm{NMR}$

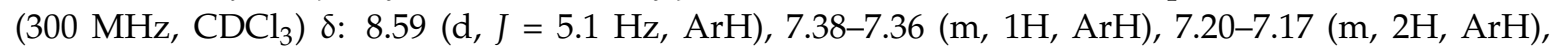
$7.07(\mathrm{dd}, J=6.6,2.4 \mathrm{~Hz}, 1 \mathrm{H}, \mathrm{ArH}), 6.88(\mathrm{~d}, J=5.1 \mathrm{~Hz}, 1 \mathrm{H}, \mathrm{ArH}), 4.08\left(\mathrm{~s}, 3 \mathrm{H}, \mathrm{OCH}_{3}\right) ; \mathrm{ESI}-\mathrm{MS}(\mathrm{m} / \mathrm{z})$ : $303.3[\mathrm{M}+\mathrm{H}]^{+}$.

4-(4'-Methylphenylamino)-8-methoxyquinoline (2g). Yield: 78.46\%; m.p. $258-260{ }^{\circ} \mathrm{C} .{ }^{1} \mathrm{H}-\mathrm{NMR}(300 \mathrm{MHz}$, $\left.\mathrm{CDCl}_{3}\right)$ 8: $8.56(\mathrm{~d}, J=5.4 \mathrm{~Hz}, 1 \mathrm{H}, \mathrm{ArH}), 7.43-7.47(\mathrm{~m}, 2 \mathrm{H}, \mathrm{ArH}), 7.22-7.17(\mathrm{~m}, 4 \mathrm{H}, \mathrm{ArH})$, $7.06(\mathrm{dd}, J=6.9,1.5 \mathrm{~Hz}, 1 \mathrm{H}, \mathrm{ArH}), 6.93(\mathrm{~d}, J=5.4 \mathrm{~Hz}, 1 \mathrm{H}, \mathrm{ArH}), 4.08\left(\mathrm{~s}, 3 \mathrm{H}, \mathrm{OCH}_{3}\right), 2.39\left(\mathrm{~s}, 3 \mathrm{H}, \mathrm{CH}_{3}\right)$; ESI-MS $(m / z): 265.4[\mathrm{M}+\mathrm{H}]^{+}$.

4-(3'-Methoxyphenylamino)-8-methoxyquinoline (2h). Yield: 80.96\%; m.p. $\quad 231-233{ }^{\circ} \mathrm{C} .{ }^{1} \mathrm{H}-\mathrm{NMR}$ $\left(300 \mathrm{MHz}, \mathrm{CDCl}_{3}\right) \delta: 8.61(\mathrm{~d}, J=5.1 \mathrm{~Hz}, \mathrm{ArH}), 7.49-7.41(\mathrm{~m}, 2 \mathrm{H}, \mathrm{ArH}), 7.32(\mathrm{t}, J=5.4 \mathrm{~Hz}, 1 \mathrm{H}, \mathrm{ArH})$, 7.13-7.10 (m, 1H, ArH), 7.06 (dd, J = 6.9, 1.8 Hz, ArH), 6.90-6.84 (m, 2H, ArH), $6.73(\mathrm{dd}, J=8.1,2.1$ $\mathrm{Hz}, 1 \mathrm{H}, \mathrm{ArH}), 4.08\left(\mathrm{~s}, 3 \mathrm{H}, \mathrm{OCH}_{3}\right), 3.83\left(\mathrm{~s}, 3 \mathrm{H}, \mathrm{OCH}_{3}\right)$; ESI-MS $(\mathrm{m} / \mathrm{z}): 281.2[\mathrm{M}+\mathrm{H}]^{+}$.

4-(4'-Isopropylphenylamino)-8-methoxyquinoline (2i). Yield: 79.47\%; m.p. $\quad 236-238{ }^{\circ} \mathrm{C} .{ }^{1} \mathrm{H}-\mathrm{NMR}$ $\left(300 \mathrm{MHz}, \mathrm{CDCl}_{3}\right)$ 8: $8.53(\mathrm{~d}, J=5.1 \mathrm{~Hz}, 1 \mathrm{H}, \mathrm{ArH}), 7.48-7.40(\mathrm{~m}, 2 \mathrm{H}, \mathrm{ArH}), 7.30-7.21(\mathrm{~m}, 4 \mathrm{H}, \mathrm{ArH})$, $7.05(\mathrm{dd}, J=6.9,2.1 \mathrm{~Hz}, 1 \mathrm{H}, \mathrm{ArH}), 6.99-6.96(\mathrm{~m}, 1 \mathrm{H}, \mathrm{ArH}), 4.08\left(\mathrm{~s}, 3 \mathrm{H}, \mathrm{OCH}_{3}\right), 2.99-2.90(\mathrm{~m}, 1 \mathrm{H}, \mathrm{CH})$, $1.29\left(\mathrm{~d}, J=6.9 \mathrm{~Hz}, 6 \mathrm{H}, \mathrm{CH}_{3}\right)$; ESI-MS $(m / z): 293.6[\mathrm{M}+\mathrm{H}]^{+}$.

4-(3'-Chloro-4'-methylphenylamino)-8-methoxyquinoline (2j). Yield: 76.48\%; m.p. $270-272{ }^{\circ} \mathrm{C} .{ }^{1} \mathrm{H}-\mathrm{NMR}$ $\left(300 \mathrm{MHz}, \mathrm{CDCl}_{3}\right) \delta: 8.61(\mathrm{~d}, J=5.4 \mathrm{~Hz}, 1 \mathrm{H}, \mathrm{ArH}), 7.47-7.41(\mathrm{~m}, 2 \mathrm{H}, \mathrm{ArH}), 7.31(\mathrm{~d}, J=2.4 \mathrm{~Hz}, 1 \mathrm{H}$, ArH), $7.25(\mathrm{~d}, J=7.8 \mathrm{~Hz}, 1 \mathrm{H}, \mathrm{ArH}), 7.12-7.04(\mathrm{~m}, 2 \mathrm{H}, \mathrm{ArH}), 6.99(\mathrm{~d}, J=5.1 \mathrm{~Hz}, 1 \mathrm{H}, \mathrm{ArH}), 4.08(\mathrm{~s}, 3 \mathrm{H}$, $\left.\mathrm{OCH}_{3}\right), 2.39\left(\mathrm{~s}, 3 \mathrm{H}, \mathrm{CH}_{3}\right)$; ESI-MS $(m / z): 299.3[\mathrm{M}+\mathrm{H}]^{+}$.

4-(2,3,4-Trifluorophenylamino)-8-methoxyquinoline (2k). Yield: 70.00\%; m.p. $238-239{ }^{\circ} \mathrm{C} .{ }^{1} \mathrm{H}-\mathrm{NMR}$ $\left(300 \mathrm{MHz}, \mathrm{CDCl}_{3}\right) \delta: 8.61(\mathrm{~d}, J=5.1 \mathrm{~Hz}, 1 \mathrm{H}, \mathrm{ArH}), 7.57-7.45(\mathrm{~m}, 2 \mathrm{H}, \mathrm{ArH}), 7.24-7.16(\mathrm{~m}, 1 \mathrm{H}$, $\mathrm{ArH}), 7.10-6.84(\mathrm{~m}, 2 \mathrm{H}, \mathrm{ArH}), 6.84(\mathrm{~d}, J=5.4 \mathrm{~Hz}, 1 \mathrm{H}, \mathrm{ArH}), 4.10\left(\mathrm{~s}, 3 \mathrm{H}, \mathrm{OCH}_{3}\right)$; ESI-MS $(\mathrm{m} / \mathrm{z})$ : $305.2[\mathrm{M}+\mathrm{H}]^{+}$.

4-(4'-Nitrophenylamino)-8-methoxyquinoline (21). Yield: 78.18\%; m.p. $265-267{ }^{\circ} \mathrm{C} .{ }^{1} \mathrm{H}-\mathrm{NMR}(300 \mathrm{MHz}$, $\left.\mathrm{CDCl}_{3}\right) \delta: 8.79(\mathrm{~d}, J=4.8 \mathrm{~Hz}, 1 \mathrm{H}, \mathrm{ArH}), 8.25(\mathrm{~d}, J=9 \mathrm{~Hz}, 2 \mathrm{H}, \mathrm{ArH}), 7.51-7.46(\mathrm{~m}, 2 \mathrm{H}, \mathrm{ArH})$, $7.37(\mathrm{~d}, J=4.8 \mathrm{~Hz}, 1 \mathrm{H}, \mathrm{ArH}), 7.29-7.26(\mathrm{~m}, 2 \mathrm{H}, \mathrm{ArH}), 7.11(\mathrm{t}, J=4.2 \mathrm{~Hz}, 1 \mathrm{H}, \mathrm{ArH}), 4.10\left(\mathrm{~s}, 3 \mathrm{H}, \mathrm{OCH}_{3}\right)$; ESI-MS $(m / z): 296.2[\mathrm{M}+\mathrm{H}]^{+}$.

4-(4'-Hydroxyphenylamino)-8-methoxyquinoline (2m). Yield: 58.17\%; m.p. $\quad 236-238{ }^{\circ} \mathrm{C} .{ }^{1} \mathrm{H}-\mathrm{NMR}$ $(300 \mathrm{MHz}, \mathrm{DMSO}) \delta: 9.44(\mathrm{~s}, 1 \mathrm{H}, \mathrm{OH}), 8.61(\mathrm{~s}, 1 \mathrm{H}, \mathrm{ArH}), 8.29(\mathrm{~d}, J=5.1 \mathrm{~Hz}, 1 \mathrm{H}, \mathrm{ArH})$, $7.89(\mathrm{~d}, J=8.7 \mathrm{~Hz}, 1 \mathrm{H}, \operatorname{ArH}), 7.38(\mathrm{t}, J=8.4 \mathrm{~Hz}, 1 \mathrm{H}, \operatorname{ArH}), 7.12(\mathrm{t}, J=8.4 \mathrm{~Hz}, 2 \mathrm{H}, \operatorname{ArH})$, $6.84(\mathrm{~d}, J=8.7 \mathrm{~Hz}, 2 \mathrm{H}, \mathrm{ArH}), 6.56(\mathrm{~d}, J=5.1 \mathrm{~Hz}, 1 \mathrm{H}, \mathrm{ArH}), 3.91\left(\mathrm{~s}, 3 \mathrm{H}, \mathrm{OCH}_{3}\right) ; \operatorname{ESI}-\mathrm{MS}(\mathrm{m} / \mathrm{z})$ : $267.3[\mathrm{M}+\mathrm{H}]^{+}$.

4-(3'-Cyanophenylamino)-8-methoxyquinoline (2n). Yield: 91.43\%; m.p. $255-256{ }^{\circ} \mathrm{C} .{ }^{1} \mathrm{H}-\mathrm{NMR}(300 \mathrm{MHz}$, $\left.\mathrm{CDCl}_{3}\right) \delta: 8.68(\mathrm{~d}, J=5.1 \mathrm{~Hz}, 1 \mathrm{H}, \mathrm{ArH}), 7.55-7.40(\mathrm{~m}, 5 \mathrm{H}, \mathrm{ArH}), 7.11-7.08(\mathrm{~m}, 2 \mathrm{H}, \mathrm{ArH}), 6.74(\mathrm{~s}, 1 \mathrm{H}$, $\mathrm{ArH}), 4.10\left(\mathrm{~s}, 3 \mathrm{H}, \mathrm{OCH}_{3}\right)$; ESI-MS $(\mathrm{m} / \mathrm{z}): 276.2[\mathrm{M}+\mathrm{H}]^{+}$.

\subsection{Cell Proliferative Assay}

The antiproliferative activities of the prepared 4-anilinoquinolines against HeLa and BGC823 cell lines were evaluated by MTT assay in vitro, with gefitinib as the positive control. The negative control contains cells, culture medium, MTT and DMSO. All human tumor cells were cultured in RPMI 1640 medium supplemented with $10 \%$ fetal bovine serum (FBS). Cells were detached by trypsinisation, seeded at 1.0-2.0 $\times 10^{3}$ cells each well in a 96-well plate and incubated in $5 \% \mathrm{CO}_{2}$ at $37^{\circ} \mathrm{C}$ overnight, then treated with the test compounds at different concentration and incubated for $96 \mathrm{~h}$. Fresh MTT 
solution was added to each well and incubated at $37{ }^{\circ} \mathrm{C}$ for $4 \mathrm{~h}$. The MTT-formazan formed by metabolically viable cells was dissolved in $150 \mu \mathrm{L}$ DMSO each well, and monitored by a microplate reader at dual-wavelength of $490 \mathrm{~nm}$; $\mathrm{IC}_{50}$ was defined as the drug concentrations that inhibited the cell number to $50 \%$ after $96 \mathrm{~h}$. Each test was performed three times.

\section{Conclusions}

In summary, two novel series of 4-anilinoquinolines were designed and synthesized as potentially potent and selective antitumor inhibitors. All of the final compounds were generated from aniline derivatives via six step reaction sequences including nucleophilic substitution, cyclization, hydroxylation, decarboxylation, chlorination and nucleophilic substitution. Among the 7-fluoro-4-anilinoquinolines, all the prepared compounds displayed some cytotoxic activity against the HeLa and BGC823 cell lines. Compounds $\mathbf{1 a}-\mathbf{g}, \mathbf{2 c}$ and $\mathbf{2 e}$ displayed superior cytotoxic activities against the BGC823 cell line than gefitinib. Furthermore, compound 1f displayed good cytotoxic activities against HeLa and BGC823 cells $\left(\mathrm{IC}_{50}\right.$ value of $10.18 \mu \mathrm{M}$ and $8.32 \mu \mathrm{M}$ against HeLa and BGC823 cells, respectively). In particular, compound $2 \mathbf{i}$ exhibited the most potent inhibitory activity against BGC 823 cells ( $\mathrm{IC}_{50}$ value of $7.15 \mu \mathrm{M}$ and $4.65 \mu \mathrm{M}$ against HeLa and BGC823 cells, respectively). The drug-like structural optimization based on the 4 -anilinoquinoline skeleton will be reported in the future.

Acknowledgments: This work was supported by the grant from the Scientific Research Project of the Department of Education of Liaoning Province (Grant No. L2013171), the Natural Science Foundation of Liaoning Province of China (Grant No. 2015020695), the Natural Science Foundation of China (Grant No. 21372156). Additionally, we thank Weiling Wang, at the Experimental Therapeutics Center, Agency for Science, Technology and Research of Singapore, for her English revisions and constructive criticism.

Author Contributions: T.L., J.K., Y.Z. and H.-F.W. performed the experiments. D.L. and T.L. designed the experiments and wrote the paper. All authors took part in data analysis and discussion. All authors read and approved the final manuscript.

Conflicts of Interest: The authors declare no conflict of interest.

\section{References}

1. Pao, W.; Miller, V.; Zakowski, M.; Doherty, J.; Politi, K.; Sarkaria, I.; Singh, B.; Heelan, R.; Rusch, V.; Fulton, L.; et al. EGF receptor gene mutations are common in lung cancers from "never smokers" and are associated with sensitivity of tumors to gefitinib and erlotinib. Proc. Natl. Acad. Sci. USA 2004, 101, 13306-13311. [CrossRef] [PubMed]

2. Bikker, J.A.; Brooijmans, N.; Wissner, A.; Mansour, T.S. Kinase domain mutations in cancer: Implications for small molecule drug design strategies. J. Med. Chem. 2009, 52, 1493-1509. [CrossRef] [PubMed]

3. Feng, Y.F.; Zeng, C.L. Advance in research for lapatinib, a dual inhibitor of EGFR and ErbB2 tyrosine kinase activity. Chin. New Drugs J. 2007, 16, 1990-1993.

4. Tao, L.Y.; Fu, L.W. Progress of study on lapatinib. Chin. Pharmacol. Bull. 2008, 24, 1541-1544.

5. Solca, F.; Dahl, G.; Zoephel, A.; Bader, G.; Sanderson, M.; Klein, C.; Kraemer, O.; Himmelsbach, F.; Haaksma, E.; Adolf, G.R. Target binding properties and cellular activity of afatinib (BIBW 2992), an irreversible ErbB family blocker. J. Pharmacol. Exp. Ther. 2012, 343, 342-350. [CrossRef] [PubMed]

6. Chen, X.; Zhu, Q.; Zhu, L.; Pei, D.; Liu, Y.; Yin, Y.; Schuler, M.; Shu, Y. Clinical perspective of afatinib in non-small cell lung cancer. Lung Cancer. 2013, 81, 155-161. [CrossRef] [PubMed]

7. Rewcastle, G.W.; Denny, W.A.; Bridges, A.J.; Zhou, H.R.; Cody, D.R.; McMichael, A.; Fry, D.W. Tyrosine kinase inhibitors. 5. Synthesis and structure-activity relationships for 4-[(phenylmethyl)amino]- and 4-(phenylamino) quinazolines as potent adenosine $5^{\prime}$-triphosphate binding site inhibitors of the tyrosine kinase domain of the epidermal growth factor receptor. J. Med. Chem. 1995, 38, 3482-3487. [PubMed]

8. Wissner, A.; Berger, D.M.; Boschelli, D.H.; Floyd, M.B.; Greenberger, L.M.; Gruber, B.C.; Johnson, B.D.; Mamuya, N.; Nilakantan, R.; Reich, M.F.; et al. 4-Anilino-6,7-dialkoxyquinolime-3-carbonitrile inhibitors of epidermal growth factor receptor kinase and their bioisosteric relationship to the 4-anilino-6,7dialkoxyquinazoline inhibitors. J. Med. Chem. 2000, 3, 3244-3256. [CrossRef] 
9. Barbosa, M.L.D.C.; Lima, L.M.; Tesch, R.; Sant'Anna, C.M.R.; Totzke, F.; Kubbutat, M.H.G.; Schächtele, C.; Laufer, S.A.; Barreiro, E.J. Novel 2-chloro-4-anilino-quinazoline derivatives as EGFR and VEGFR-2 dual inhibitors. Eur. J. Med. Chem. 2014, 71, 1-14. [CrossRef] [PubMed]

10. Jiang, N.; Zhai, X.; Zhao, Y.F.; Liu, Y.J.; Qi, B.H.; Tao, H.Y.; Gong, P. Synthesis and biological evaluation of novel 2-(2-arylmethylene) hydrazinyl-4-aminoquinazoline derivatives as potent antitumor agents. Eur. J. Med. Chem. 2012, 54, 534-541. [CrossRef] [PubMed]

11. Minami, Y.; Shimamura, T.; Shah, K.; LaFramboise, T.; Glatt, K.A.; Liniker, E.; Borgman, C.L.; Haringsma, H.J.; Feng, W.; Weir, B.A.; et al. The major lung cancer-derived mutants of ERBB2 are oncogenic and are associated with sensitivity to the irreversible EGFR/ERBB2 inhibitor HKI-272. Oncogene 2007, 26, 5023-5027. [CrossRef] [PubMed]

12. Wissner, A.; Mansour, T.S. The development of HKI-272 andrelated compounds for the treatment of cancer. Arch. Pharm. 2008, 341, 465-477. [CrossRef] [PubMed]

13. Pawar, V.G.; Sos, M.L.; Rode, H.B.; Rabiller, M.; Heynck, S.; Otterlo, W.A.L.V.; Thomas, R.K.; Rauh, D. Synthesis and biological evaluation of 4-anilinoquinolines as potent inhibitors of epidermal growth factor receptor. J. Med. Chem. 2010, 53, 2892-2901. [CrossRef] [PubMed]

14. Roskoski, R., Jr. The ErbB/HER family of protein-tyrosine kinases and cancer. Pharmacol. Res. 2014, 79, 34-74. [CrossRef] [PubMed]

15. Zhou, S.Z.; Li, X.H.; Zhang, Y.X. Methylation patterns of the human EGFR gene promoter region. Chin. J. Biochem. Mol. Biol. 2010, 26, 568-574.

16. Long, H.; Li, H.; Wu, Q.M. RNA interference targeting EGFR on proliferation and apoptosis of human gastric carcinama cell line BCG823. China J. Mod. Med. 2012, 22, 59-62.

17. Ray, A.W.; Joseph, C.M.; Victor, K.E.; Phil, J.G.; George, S.R. Quinoline, Quinazoline, and Cinnoline Fungicides. E.P. Patent 0,326,330(A2), 2 August 1989.

Sample Availability: Not available.

(C) 2015 by the authors; licensee MDPI, Basel, Switzerland. This article is an open access article distributed under the terms and conditions of the Creative Commons by Attribution (CC-BY) license (http://creativecommons.org/licenses/by/4.0/). 